\title{
Collaborative Optimization Analysis on GSM/WLAN and TD-S/TD-L network
}

\author{
Bao GUO ${ }^{1, \text { a }}$, Jin-Jian Zhang ${ }^{2, \text { b }}$, Yang ZHANG ${ }^{3, c}$ \\ ${ }^{1,2}$ CMCC Shanxi Co., Ltd. Taiyuan Branch, Taiyuan, China \\ ${ }^{3}$ Dept. of Networks, CMCC, Beijing, China \\ aguobao@sx.chinamobile.com, \\ bzhangjinjian@sx.chinamobile.com, \\ 'zhangyangwl@chinamobile.com
}

Keywords: collaborative traffic efficiency

\begin{abstract}
Mobile operators faced with rapid growth in data throughput, However, the rapid growth of traffic from $2 \mathrm{G}$ networks led to the $2 \mathrm{G}$ network load increases, even affect the quality of voice communications. At the same time, TD-SCDMA network is relatively light load and WLAN network is limited by the terminal and the hot spots, they can't give users a seamless broadband data services. It is discussed in this article on how to quadruple collaboration and flow of business to make the optimization analysis from the user traffic bandwidth requirements for different types of data services.
\end{abstract}

\section{GSM/WLAN and TD-S/TD-L network collaborative background}

Along with the rapid development of mobile Internet, the demand rises for mobile data services and the growth of mobile data traffic is rapid. On point of view of user, although in recent years, traffic tariff packages declines, but the users of mobile Internet are still a light application of low-flow-based. Traffic flow below $50 \mathrm{M}$ users accounted for over $70 \%$, and tariff is still a major obstacle restricting user access internet by mobile phone.

Mobile data traffic is mainly concentrated in the $2 \mathrm{G}$ and WLAN networks. Radio channel resource occupied by the data services is more than voice services in $2 \mathrm{G}$ network. Date service swallowed a large number of wireless channels in some areas, which causes voice services can only use half rate, affecting the quality of the call. Application packet of lots of instant messaging services is small, but also consumes the same resources like browsing etc. It causes precious radio channel resource waste as well as data services carrying inefficient.

WLAN is difficult to form a continuous coverage due to technical limitations, it is limited to hot spots, lack of unified planning, At present, the WLAN network traffic behaves rapid growth but fewer business users.

TD-SCDMA network in urban areas has achieved continuous coverage, but due to the constraints of terminal, the user perception is unsatisfactory. In addition, the TD test terminal average received power level is higher than commercial terminals about $10-14 \mathrm{~dB}$. It causes the roads and regional continuous coverage in accordance with the test terminal, in the user's actual use of the process, but a large number of weak coverage points, led to the use of business occurred during the frequent interoperability serious impact on the use of perception.

Also, TD-SCDMA network optimization process, the operator too much emphasis on targets, leading to equipment manufacturers set unreasonable $23 \mathrm{G}$ interoperability threshold, so the TD terminal re-election as soon as possible, switch to the $2 \mathrm{G}$ network.

A number of reasons led to the TD network utilization is too low, part of the new TD terminal still reside in the $2 \mathrm{G}$ network, the objective to increase the pressure on the already overburdened $2 \mathrm{G}$ networks. The LTE network is still in the pilot phase of scale. Therefore, a need to standardize consider four network synergies to meet the needs of the users different levels of wireless data services. 


\section{GSM network to be optimized to enhance the carrying efficiency}

$2 \mathrm{G}$ network traffic sustained rapid growth in recent years, data services loads is under increasing pressure. Due to the EGPRS technical limitations, China Mobile faced with the strong impact of data services of other operators.

With the rapidly growing popularity of intelligent terminals, mobile QQ and micro-blogging services are favored by more and more users, instant messaging service only apply a small packet, but the network according to its terminal type, assign the same radio time slot as browsing services. It cause a greater waste, which lead to the lower bearing efficiency of data services.

EGPRS is theoretically possible to achieve wireless transmission rate of $59.2 \mathrm{kbps}$, a provincial capital city $2 \mathrm{G}$ networks actually carrying efficiency the only $3.86 \mathrm{kbps}$, there is a big gap with the theoretical value.

In addition, the low efficiency of the data service occupies about $60 \%$ of the radio channel resources, it causes a large number of voice services using the half rate, affects call quality. EGPRS network is urgent to enhance the data channel carrying efficiency to meet customers' business needs, at the same time, to provide voice services with adequate resources, to reduce the noise interference of the network to improve call quality.

To improve the the EGPRS coverage to enhance the efficiency of the data service bearer EGPRS wireless transmission rate is $59.2 \mathrm{kbps}$ theory, GPRS CS-2 coding rate is commonly used as a $13.4 \mathrm{kbps}$, large difference between the two. Thus, to enhance the EGPRS coverage can improve efficiency of data service bearer.

EGPRS coverage rate of December road test in a city is average of $96.92 \%$, but EGPRS coverage one grid for $89 \%$ only. There are two main reasons for low EGPRS coverage after analysis, first: PCU_B and PCU2_D (NSN equipment) is mixed insertion; Second SEGMENT (NSN equipment a logical plot, private access supported EDGE Terminal) is serious congestion in the cell, too many access applications, maximum reuse of up to $9 \mathrm{TBF} / \mathrm{TSL}$ (can gain access to the maximum value), resulting in Terminal cannot access EDGE BTS and use GPRS BTS.

For PCU mixed into this situation, because the processing capacity of the PCU2_D board is twice times of PCU_B, it is an ideal optimization measures to replace the PCU_B with the PCU2_D board. If you cannot replace the board timely, PCU type where the grids in the cell are needed for statistics, will be vested in the cell involving Terminal flow adjustment under PCU_B under PCU2_D, so when in the road test, the test terminals occupy the district in PCU2_D board, to avoid due to take up the case of GPRS cell reselection between the different boards. In addition, indoor coverage for cell or that does not affect the flow road coverage under adjustments to PCU_B, maintain a different Board of load balancing, avoid hanging under the PCU2_D too many adverse effects in the cell.

For EDGE BTS congestion caused subscribers access GPRS BTS, to meet the voice, a busy residential expansion of the carrier frequency or cell division. If the expansion is difficulty that the SEGMENT mode may be temporarily changed back to residential EDGE / GPRS mixed mode.

PCU mixed insertion problem resolved, the EDGE coverage has upgraded from $89 \%$ to $99.9 \%$, improving significantly. EDGE BTS congestion problems after optimizing, EDGE BTS complex expense has decreased from 7.88 to 3.94 and GPRS BTS multiplexing has decreased from 6.3 to 3.15 , the maximum reuse has significantly declined. Field testing indicators display the EGPRS coverage than the upgrade from $95.47 \%$ to $99.2 \%$. Optimization effect is obvious.

\section{To open the packet detection to enhance the efficiency of the data service bearer}

Gn interface (interface between SGSN and GGSN) statistics show that small flow packet is a high percentage of business $2 \mathrm{G}$ network. Less than 1024B packets is accounting for $69 \%$. Open the packet detection can effectively enhance the efficiency of GPRS bearer. Packet detection is based on the length of the PDU.

If the length of the PDU does not exceed a certain threshold (250byte) the default in accordance with the single time slot is allocated when the PDU is longer than the packet door limit will immediately be restored in accordance with the ability of mobile terminal multi-slot allocation mechanism. 
Packet detection feature in the current network BSC112 for authentication, as shown in Figure 1, compared to open before and after, the PDCH carrying efficiency of each timeslot has improved significantly, especially when the data services is the most busy at 22:00, it is a 28.5 percent increase from 5.62 to 7.22. In addition, in the actual comparison of the test, open packet detection during the ping test BSC, BSC when the packet is less than 250Byte users are assigned a time slot, when the packet is 250Byte BSC 4 time slots allocated for it.

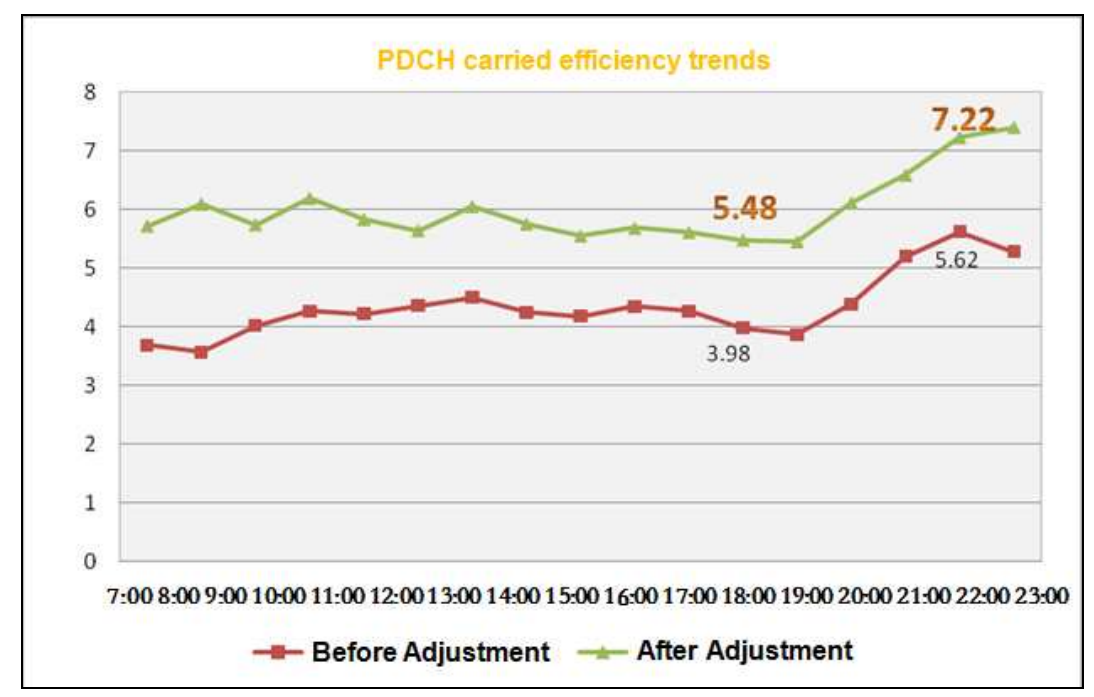

Figure 1: "Packet inspection" on PDCH carried efficiency trends

\section{The main problem of the TD network and optimization measures}

At present the assessment indicators of good TD network, excellent automatic drive test ATU test indicators, but the user's perception is poor, there are lots of VIP users' complaints.

This is a popular commercial terminals (Samsung 19108) and test terminals (Tang 8142) comparison test in the same car to see the contrast from the reception level, commercial terminals' receive level is weaker than test terminals about $10-14 \mathrm{dBm}$, as shown in Figure 2. The TD-SCDMA network planning of the city is all in accordance with the test terminal to complete a continuous coverage, but in the user's actual use of the process, there are a lot of weak coverage, which seriously affects the quality of the call, causes frequent $23 \mathrm{G}$ interoperability.

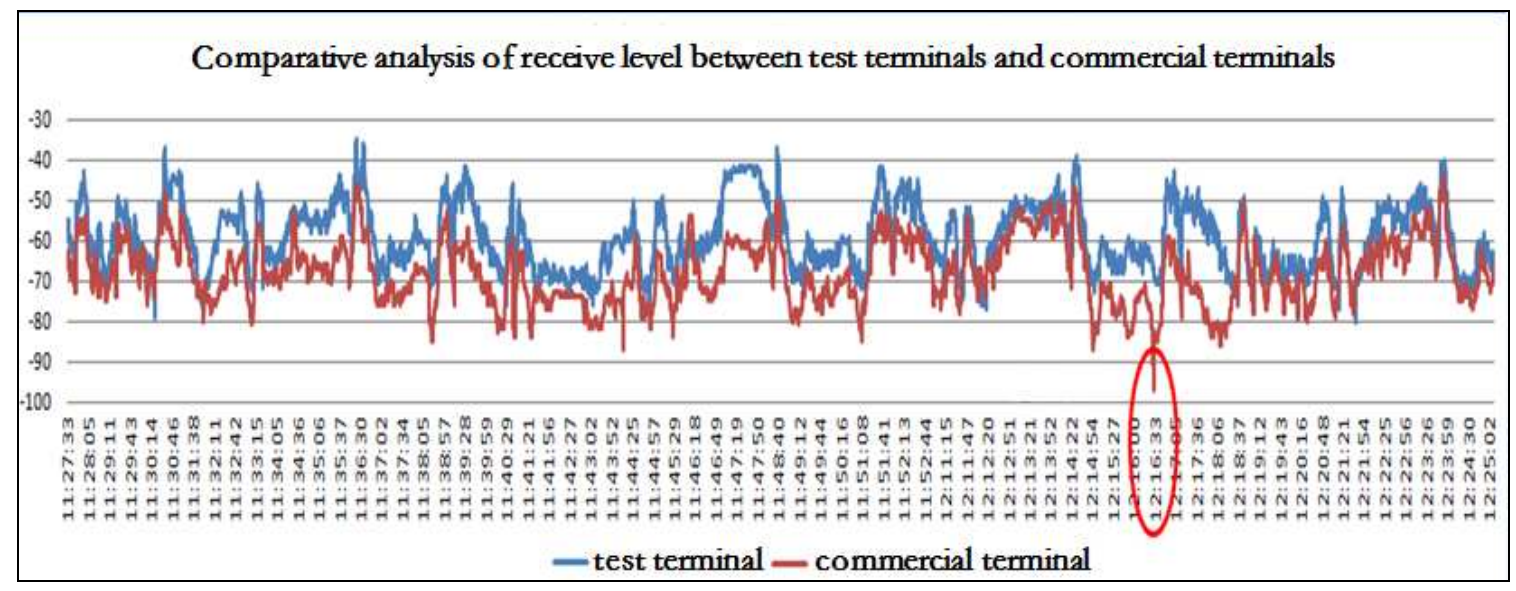

Figure 2: The test terminals and commercial terminals to receive level

\section{Optimization of power parameters and antenna tilt angle to enhance the coverage}

Determine the existing TD-SCDMA network is a continuous coverage is inadequate, within the scope of the entire network to raise the general base station transmitting power and adjust the antenna tilt angle combined with optimization to improve TD coverage. Include: 
1, Adjust the base station transmitting power settings. In accordance with the RRU model to recalculate the maximum transmit power of each cell, re-planning PCCPCH channel power setting. 2, Adjust the azimuth and elevation angle. Direction by adjusting the antenna azimuth optimization district coverage by step uplift district pitch angle to enhance coverage. View of the TD-SCDMA as the self-interference system is adjusting the pitch angle at the same time, concerned about the changes in cell interference, and frequency / scrambling code optimization to suppress interference enhancement.

\section{G adjacent areas optimized to enhance the TD data traffic}

In order to ensure that users perceive, in the TD signal is weak cannot guarantee that the user's communication, the successful and timely switch to the GSM network. TD side add to the GSM network adjacent areas, the TD signal better ensure that the user communication, the user goes back to $3 \mathrm{Gnetwork}$ the presence of the $2 \mathrm{G}$ network perfect add $3 \mathrm{G}$ adjacent areas is critical. If the $2 \mathrm{G}$ cell is not added TD adjacent areas, then the TD switch to the $2 \mathrm{G}$ cell, resident for some time, until the re-election to add a TD adjacent areas of the $2 \mathrm{G}$ cell, in order to initiate the re-election to the TD-SCDMA network, if it time to initiate the data services, then the user's perception cannot be fully guaranteed.

Take Taiyuan City for example, TD's $2 \mathrm{G}$ adjacent cells are the only new station part of the plot missing $2 \mathrm{G}$ adjacent areas. There are some unreasonable adjacent cells such as $1132 \mathrm{GSM}$ cells have no TD adjacent cells in the cell and 1300 GSM cells' TD adjacent cells is less than 2. $2 \mathrm{G}$ district of the region's overall planning and optimization of TD adjacent cells after the completion of the adjacent areas of ZTE region $2 \mathrm{G}$ district added a total of 29,062 TD adjacent cells. In order to ensure the user's perception, from $2 \mathrm{G}$ re-election back to the TD parameter is set back to the T network when the TD level greater than-84dbm. After optimization of the wireless parameter of re-election, the data traffic of TD network show significant growth, as shown in Figure 3.

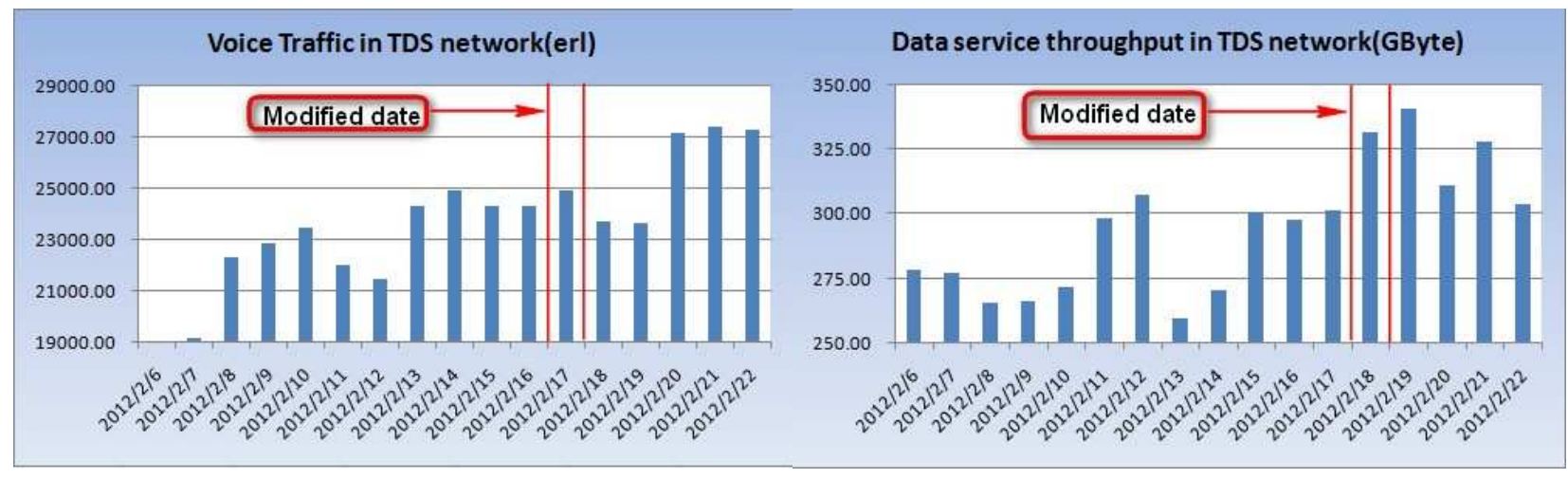

Figure 3: TD data traffic and system optimization to increase the $2 \mathrm{G} T \mathrm{TD}$ adjacent areas the number of re-election

\section{The coverage optimization based on TD Commercial terminal}

Large differences in test terminals and commercial terminals, resulting in a continuous coverage of the TD network in accordance with the test terminal, the user is not continuous, weak coverage, seriously affected the perception. Resolve user complaints process, the optimization engineers often use test Terminal for field testing, and test results are not representative of the reality of the user, thus leading to the optimization engineers think the coverage has been resolved, but the user is not authorized.

Through extensive road testing in the near future, discovered a weak business terminal cover many issues. A typical weak coverage areas are found East of YingZe bridge, was one of the more open areas in the region, including the North ring road, South of YingZe bridge ramp and YingZe bridge, large, complex wireless bridge and ramp environment, weak business Terminal covers issues very seriously. After analysis there are two ways to solve the problem:

1, increase a cell strengthened the existing Chu-Lin Park site of YingZe bridge East, North on-ramp of yingze bridge cover; 
2, Pearl Catherine Park of new 3 cell and 1 cell merged, merged the two antenna-feeder system covering not only ensuring coverage and reduced the cell switching edge of weak field.

The cell coverage of the base station "Pearl Park" is a fast-moving scene, both in or near the East road of YingZe Bridge. most of the users are users who move faster, therefore, switch targets on user perception of a great Association. After optimal adjustment, especially the merger of the new residential and a plot of the original beads Lynn Park, the switch performance has been improved significantly, switch system, the success rate increased to 99.7 from 91.4 percent, to enhance by about 7 percentage points, the effect is very obvious.

\section{WLAN, LTE collaborative optimization measures}

WLAN networks exist a problem at present as "more construction and less use", for example, there is a statistic show that existing WLAN hot pot 2,799, AP 28,974, but statistic found that low traffic hot spots is ratio of $67.02 \%$ in late March 1876 , accounting for $69.1 \%$. The analysis found that: WLAN site planning methods: In accordance with $2 / 3 \mathrm{G}$ high flow cell, and with reference to market demand to plan. The $2 / 3 \mathrm{G}$ district polygon coverage, WLAN hotspot is the point-like cover, this degree of coincidence coarse, cannot be precisely targeted to the real needs of the physical location or building.

Through traffic analysis on the business user data and found flow big does not mean users, network construction to meet users ' demands as a precondition. WLAN hotspot planning required from the viewpoint of the user needs, development of appropriate rules, analyze and judge flow relatively static user location, lock the regional demand for WLAN area. And mathematical modeling approaches, WLAN planning point positioning in the context of a more definite.

Secondly, you need to consider the advantages of WLAN network, WLAN can support broadband mobile data, can be very well supported by data bandwidth is very large, but very small business the income, such as downloads, browsing, P2P video business.

Finally, the choice of site and marketing of the WLAN can also be intelligent terminals, data cards, Internet this such terminal, the terminal is not limited to support WiFi terminal.

Standard LTE OFDMA and MIMO can support 20MHz provides downlink spectrum bandwidth $100 \mathrm{Mbps}$, uplink peak rates of 50Mbps; to improve the performance of users cell edge, to increase cell capacity, and to reduce system latency, user plane internal one-way transmission delay less than $5 \mathrm{~ms}$, the control plane from sleep to active migration time less than $50 \mathrm{~ms}$, from reside status to active migration time less than 100ms. China LTE trial network is in the stage, China Mobile integration, and actively explore the TDD-LTE and FDD-LTE technology to provide users with broadband mobile data services.

\section{Summary}

China Mobile will have to face the situation of the GSM / TD-SCDMA / WLAN / TD-LTE network coexistence, to replace the law and not follow the traditional industry the $2 \mathrm{G} / 3 \mathrm{G} / 4 \mathrm{G}$ between the network life cycle. How to take into consideration the format differences between the four networks, technical characteristics, load capacity, factors such as maturity of technology development, enables the four networks to unified collaborative operations, is a long time in the future must face and take the problem seriously.

\section{About the author:}

Guo Bao, Bachelor of Shanxi College and Master of science in engineering of North-Central University, Shanxi mobile level II expert, long-term engaged in wireless network optimization work, has rich of line work experience, has independent is responsible for in Taiyuan of GPRS, and TD wireless optimization work, has authorized national invention patent a items, and application national invention patent four items, and published one monograph "TD-SCDMA Wireless network planning and optimization", over published various technology papers more than 40 papers. 


\section{References}

[1] Li Shihe: TD-SCDMA third generation mobile communication system standard, edited by People's Posts \& Telecom, Beijing(2003), in press.

[2] TS 25.224 the Physical layer procedures (TDD)

[3] TS 25.301 Radio interface the protocol architecture

[4] Li XW, Li Guiyong: TD-SCDMA third generation mobile systems, signaling and achievement, edited by People's Posts \& Telecom, Beijing(2003), in press.

[5] Wang Yanjun: WLAN accurate coverage of the shunt the $2 / 3 \mathrm{G}$ data business program [J]. Network communications industry, 2011. 